\title{
Vitamin D concentration, body mass index, ethnicity and SARS-CoV-2I COVID-19: initial analysis of the first- reported UK Biobank Cohort positive cases (n 1474) compared with negative controls (n 4643)
}

\author{
A.L. Darling ${ }^{1}$, K. R. Ahmadi ${ }^{1}$, K.A. Ward ${ }^{2}$, N.C. Harvey ${ }^{2-3}$, A. Couto Alves ${ }^{1}$, \\ D. K. Dunn-Walters ${ }^{1}$, S.A. Lanham-New ${ }^{1}$, C. Cooper ${ }^{2-4}$ and D.J. Blackbourn ${ }^{1}$ \\ ${ }^{1}$ School of Biosciences and Medicine, University of Surrey, Guildford. GU2 $7 \mathrm{XH}$, \\ ${ }^{2}$ MRC Lifecourse Epidemiology Unit, University of Southampton, Southampton, SO17 1BJ, \\ ${ }^{3}$ NIHR Southampton Biomedical Research Centre, University of Southampton and University Hospital Southampton \\ NHS Foundation Trust, Southampton, SO16 6YD and \\ ${ }^{4}$ NIHR Oxford Biomedical Research Centre, University of Oxford, Oxford, OX4 $2 P G$
}

There is an urgent need to assess risk factors affecting individual likelihood of contracting the novel SARS-CoV-2 virus, and development of COVID-19. One potential risk factor is vitamin D deficiency ${ }^{(1)}$. Vitamin D is important for immune defence against pathogens as it promotes macrophage antimicrobial responses and the regulation of antigen-presenting dendritic cells and suppressor T-cells ${ }^{(2)}$. However, it is unclear whether $25(\mathrm{OH}) \mathrm{D}$ concentration is associated with risk of contracting SARS-CoV-2 and/or developing COVID-19.

We aimed to assess whether there is an association between serum 25(OH)D status and odds of testing positive for SARS-CoV-2, when controlling for confounders such as body mass index (BMI) and ethnicity.

We used data from the UK Biobank, including n 1474 SARS-CoV-2 positive cases and n 4643 SARS-CoV-2 negative controls. The UK Biobank study was originally approved by the UK North West Multi-Centre Research Ethics Committee (MREC); application $11 / \mathrm{NW} / 0382$, with written informed consent obtained from subjects.

Results are presented as median (interquartile range; IQR). Statistical significance was assessed by Mann-Whitney Test. Age (years) was $58.0(18)$ in cases and in $60.0(15)$ in controls $(\mathrm{P}=0.002)$. Serum $25(\mathrm{OH}) \mathrm{D}$ status was almost identical in cases $(43.3(31.0) \mathrm{nmol} / \mathrm{L})$ and controls $(44.5(30.4) \mathrm{nmol} / \mathrm{L})(\mathrm{P}=0.34)$. Serum $25(\mathrm{OH}) \mathrm{D}$ status was lower in those with obesity $(39.4(28.7) \mathrm{nmol} / \mathrm{L}) \mathrm{compared}$ with those under or normal weight $(48.0(32.2) \mathrm{nmol} / \mathrm{L})(\mathrm{P}<0.001)$. Also, 25(OH)D concentration was lower in those of Asian, Black and Mixed ethnicity $(29.1(21.8) \mathrm{nmol} / \mathrm{l})$ compared with White ethnicity $(45.6(30.2) \mathrm{nmol} / \mathrm{L})(\mathrm{P}<0.001)$.

Logistic regression model results (n 5303) are presented as odds ratio (OR) and $95 \%$ confidence interval. Variables were entered as one block so all estimates are confounder adjusted. Serum $25(\mathrm{OH}) \mathrm{D}$ concentration $(<=50 \mathrm{nmol} / \mathrm{L}$ vs

$>50 \mathrm{nmol} / \mathrm{L})$ was not associated with odds of testing positive (1.03, 0.90 to 1.18$)$. However, being obese (1.22, 1.02 to 1.44$)$; having higher deprivation index (1.25, 1.09 to 1.43$)$; living outside London (0.80, 0.66 to 0.96 ); being male (1.24,

1.09 to 1.42 ) or of Asian, Black or Mixed Ethnicity (2.0, 1.59 to 2.51) was associated with a higher odds of testing positive. Being over 70 years old $(0.82,0.71$ to 0.94$)$ or a regular smoker $(0.73,0.58$ to 0.93$)$ was associated with reduced odds.

We found no association between $25(\mathrm{OH}) \mathrm{D}$ status and odds of testing positive for SARS-CoV-2. However, the sample on average was not severely vitamin $\mathrm{D}$ deficient and results may differ in populations with $25(\mathrm{OH}) \mathrm{D}<25 \mathrm{nmol} / \mathrm{L}$. As the number of UK Biobank cases increases, we will expand our model to assess the association between $25(\mathrm{OH}) \mathrm{D}$ concentration and COVID-19-attributed mortality and symptom severity.

This research was conducted using the UK Biobank (application 15168). KAW, NCH, DKDW and CC are not collaborators on this application and did not view the individual participant data.

\section{References}

1. Lanham-New et al. (2020) Br Med J, doi:10.1136/ bmjnph-2020-000089.

2. Hewison M (2012) Proc Nutr Soc, 71, 50-61. 